\title{
Cooperative Equilibrium in Biosphere Evolution: \\ Reconciling Competition and Cooperation in Evolutionary Ecology
}

John Herring

ORCID: 0000-0002-5016-7947

Email: herring3@purdue.edu

West Lafayette, Tippecanoe County, IN, USA (Purdue University) 47906 


\section{Declarations:}

Acknowledgements: Special thanks belong to Dr. F. Jacobs and to two anonymous reviewers for their insightful and helpful criticism, and also to Benjamin Culver and Pietro Stroik for their support. This work would have been impossible without access to the Purdue University Library Database, for which the author is very grateful. The author does not commit any of those acknowledged to adherence to the views expressed in this paper. AMDG.

Conflicts of Interest: The author declares that he has no conflict of interest.

Funding: This essay was not funded by any organization or individual. The author did not make use of any University funding or assistance aside from Purdue University database access, library access, and other educational opportunities afforded by the University generally. This project is not affiliated directly with Purdue University, and the author retains responsibility for this essay.

Availability of data and material: All materials cited are available in academic databases, university libraries, or free online sites, as of the time of writing, or may be purchased. Some original source materials were unavailable to the author, who was thus obliged to rely on direct or indirect citations garnered from other sources, as outlined in the text and references.

Code Availability: not applicable

Authors' Contributions: not applicable

Ethics Approval: not applicable

Consent to Participate: not applicable

Consent for Publication: not applicable 


\begin{abstract}
:
As our understanding of biological evolution continues to deepen, tension still surrounds the relationship between competition and cooperation in the evolution of the biosphere, with rival viewpoints often associated with the Red Queen and Black Queen hypotheses respectively. This essay seeks to reconcile these viewpoints by integrating observations of some general trends in biosphere evolution with concepts from game theory. It is here argued that biodiversity and ecological cooperation are intimately related, and that both tend to cyclically increase over biological history; this is likely due to the greater relative stability of cooperation over competition as a means of long-term conflict resolution within ecosystems. By integrating this view of the biosphere with existing models such as Niche Game Theory, it may be argued that competition and cooperation in ecosystems coexist at equilibria which shift preferentially towards increasing cooperation over biological history. This potentially points to a state of "cooperative equilibrium" as a limit or endpoint in long-term biosphere evolution, such that Black Queen and Red Queen behavior dominate different phases in an evolutionary movement towards optimal cooperative stability in ecological networks. This concept, if accepted, may also bear implications for developing future mathematical models in evolutionary biology, as well as for resolving the perennial debate regarding the relative roles of conflict and harmony in nature.
\end{abstract}

Keywords: [cooperation, competition, evolution, symbiosis, biodiversity, equilibrium] 


\section{Introduction}

In the course of evolutionary development, it seems that two endmember strategies are possible on the continuum of ecological dynamics. On one hand, organisms can exist in a state of conflict, continually and even violently competing for resources, and thereby evolving and maintaining their populations at a sustainable level; this view is sometimes associated with van Valen's Red Queen hypothesis of evolutionary biology (van Valen 1973; Markos and Svorcova 2019; Sachs and Hollowell 2012; Harvey et al. 2014). Alternatively, ecological communities can adapt and find ways of interacting with other organisms for their mutual benefit, dividing and sharing available resources through complementary evolution, thus maintaining a stable biosphere; this view has more in common with the Black Queen hypothesis of Morris et al. (Morris et al. 2012; Morris et al. 2011; Sachs and Hollowell 2012). Few modern researchers would likely advocate the unqualified acceptance of either of these viewpoints, and over the past century a strong a priori emphasis on "Red Queen" competition as the primary driver of evolutionary change and biological behavior has gradually shifted toward a more nuanced perspective (Agrawal et al. 2007), as competition-centered paradigms of evolutionary biology have increasingly come under attack by numerous authors (such as Margulis, Lovelock, Roughgarden, and others; see for example Kropotkin 1902; Onori and Visconti 2012; Vermeij and Roopnarine 2013; Roughgarden 2009; Markos and Svorcova 2019; Mitteldorf 2012).

Nonetheless, it remains important to examine the tension between these competitive and cooperative views of evolutionary biology more closely, particularly with respect to the evolution of diversity (Ramos and Schiestl 2019) and its relationship to cooperation and competition across the biosphere. Many researchers have made significant contributions in this area, especially in fields such as epigenetics, theoretical biology, complexity theory, biological 
game theory, and biosemiotics, and much of their work has been integrated into the Extended Evolutionary Synthesis (Markos and Svorcova, 2019). In a number of these works, the complexity of ecosystems and their evolution has been attributed to interacting feedbacks between cooperation and competition within ecological communities (Agrawal et al. 2007, Fort 2020), and this essay seeks a balanced perspective in this rich and sometimes confusing debate. Rather than siding with a definite hypothesis, it seeks to resolve tensions between rival viewpoints in evolutionary biology, and to exhibit the implications of this rapprochement. Before exploring the resolution of this tension between the "Black Queen" and "Red Queen" hypotheses, it will consider the relationship between diversity, complexity, and cooperation in biosphere evolution, along with recent work in modeling ecosystem balance and niche game theory (Fort 2020; Fort 2008).

\section{Conflict, Competition, and Cooperation}

It is first necessary to define the meaning of some key terms (as used in this paper). Biological game theory models commonly describe ecological interactions in terms of a "+ / - " notation, such that $(+)$ indicates a positive or cooperative interaction and (-) indicates a negative or conflict-based interaction (Fort 2020, Fort 2008). For instance, positive mutualism and bilateral cooperation between organisms or species is treated as a " $+/+"$ interaction, while pure competition or conflict is a "-/_"“ interaction. Predation, parasitism, and other biological strategies constitute "+/ - " relationships, whose intricate "game" dynamics may manifest both individually competitive and communally stabilizing interactions. This essay will roughly rely on these categories, while refining the definitions of some of the key relationships discussed.

Conflict is here used in the sense of purely negative interactions (-/-), in which neither organism involved benefits from the exchange of mutual injury. This potentially unstable 
ecological configuration may evolve toward "predator-prey" relationships (differential competition) or achieve balance in a stable coexistence of competing populations, as often predicted by Lotka-Volterra models (e.g. Ackland and Gallagher 2004; Hening and Nguyen 2018; and many others). However, Lotka-Volterra competitive coexistence is unlikely to remain at a constant equilibrium level of competition throughout all of evolutionary history; rather, it is argued below that equilibrium levels of conflict tend to decrease over long timescales even in the most stable competitive systems, as initially competing populations evolve subtle interdependencies through complementary adaptation and differentiation, often mediated by the coevolving ecosystem around them. Such preferential change may be rapid in periods of instability, and extremely slow or even absent under static conditions, but as coexisting communities dynamically evolve together, the competitive relationships among a set of populations may very gradually approach increasingly stable and cooperative equilibria. Conflict among species may thus define "competitive disequilibrium" processes in biosphere evolution, so called because they do not typify optimal endpoints approached by long-term processes of ecological integration, but rather show a preferential movement toward a more integrated and cooperative equilibrium state within ecosystems. "Cooperative equilibrium" is here defined as the hypothetical long-term equilibrium state where conflicts between initially competing organisms are mutually resolved through cooperation and communication; this ideally results in a stable ecological configuration in which interacting populations work together to optimize their survival conditions communally over the long term, thereby minimizing competitive instability (for more about ecosystem equilibria including both competition and cooperation, see recent work on Niche Game Theory (Fort 2020; Fort 2008)). 
Ecological cooperation is an extremely broad term, and attempts to restrict its meaning have typically been unsuccessful (Stachowicz 2011). While pure conflict between organisms often ends in mutual destruction, the term "cooperation" is based on the Latin root roughly meaning "to work together"; this implies the relationships among a set of individuals working towards or otherwise approaching an end state of harmony from which all experience some benefit individually and as part of an integrated community. Harmony (as I briefly define it here) may be thought of as the state where oppositions are reconciled mutually and constructively, in which all parts of a system are integrated to maintain an interactive balance or submit to an ordering principle. Ecological harmony is based upon communication between different organisms and communities, and this communion or "mutual understanding" through shared communication pathways among these populations leads to greater cooperation within the total ecosystem (Markos and Svorcova 2019), while enhancing vitality and adaptivity within the biosphere as a whole. It can also play a complex evolutionary role; for instance, microbiological research by Morris et al. indicates that mutual exchange of useful metabolic byproducts, followed by discarding redundant metabolic pathways in favor of differentiated production and sharing of needed resources, may have been critical in the evolution of symbiotic and cooperative communities among prokaryotes, and potentially their eukaryotic descendants (Morris et al. 2011; Morris et al. 2012; Guerrero and Berlanga 2016; Sachs and Hollowell 2012). Ultimately, the organisms in an ecosystem can interact in mutually beneficial ways directly through symbiosis or indirectly through the mediation of shared symbionts, overlapping niches, and other interactions rippling through the ecosystem; indeed, one who extends the concept of symbiosis to the whole biosphere can find such cooperative relationships "nearly everywhere" (Markos and Svorcova 2019). 


\section{Preferential Evolution of Ecological Cooperation}

It is here argued that cooperation tends to increasingly pervade ecosystems as the biosphere evolves, developing in an intimate relationship with the evolution of complexity across all ecological levels. In general, biological complexity and biodiversity exhibit a tendency to increase over time, reducing conflict between populations and allowing mutual relationships like symbiosis to develop and extend in scope. The macroscale effects of such growing diversity, mutual adaptation, and communication across the biosphere tend to unite organisms and ecosystems in a state of "metasymbiosis". These growing cooperative relationships in turn can act to increase biological and ecological diversity (Fort 2020; Prosdocimi et al. 2020), thus completing a positive feedback cycle with strong effects on biosphere evolution. Though these processes of qualitative evolution are tightly intertwined, forming feedback loops with each other and with related evolutionary processes, it may be helpful to investigate how each of these develops across evolutionary history.

\section{Diversification and Symbiosis Across the Biosphere}

At this time, it is fairly widely accepted that evolutionary processes tend toward greater biological complexity, and hence greater diversity, in ecosystems and in relationships among organisms (Ekstig 2015; Lineweaver et al. 2013; Bonner 1988), though biological complexity has consistently proved difficult to define (Ekstig 2015; Lineweaver et al. 2013; for details about evolution of complexity, see Bonner 1988). In general, greater biodiversity provides complex systems of ecological redundancy and interwoven niche networks, thereby acting as a more effective buffer against conflict and instability and affording more protection for local communities than a simpler ecosystem can (Guerrero and Berlanga 2016; Harvey et al. 2014; Markos and Svorcova 2019). Moreover, increasing biodiversity facilitates the exploration of 
new econiches and relationships within evolving ecosystems, reducing conflict for shared resources and allowing symbiosis and other mutualisms to flourish between diverse organisms.

The importance of diversification in reducing conflict across the biosphere is not limited to ecosystems; ecological niche construction (Odling-Smee et al. 2003) manifests this process on a species or even sub-species level. While adaptive radiation periodically leads to the development of new niches within an ecosystem, such spaces in the continuum of ecological relationships are often conserved and convergently colonized by widely disparate phylogenetic groups across geologic time (McGhee et al. 2011). Within this process, complementary adaptation of different populations sharing the same econiche allows sub-niche differentiation to occur, enabling similar or convergent species to reduce competition for resources while still occupying the same primary niche. An empirical example of this might include convergently evolved niche-sharers such as butterflies and hummingbirds which, while both exhibiting similar trophic strategies and morphologies (McGhee et al. 2011), are seldom seen to directly compete for the resources on which both survive, as their metabolic requirements and specific nectar sources are significantly different. A more theoretical framework for understanding the crucial role of symbiosis and facilitative relationships in niche construction is provided by Niche Game Theory (Fort 2020), which uses mathematical biology to effectively model niche construction between competing and cooperating organisms.

Thus, direct conflict between increasingly diverse populations generally decreased as new ecological relationships were built up over the course of life history. Given the limited number of econiches available at the beginning of biological history, rising levels of competitive stress would likely drive the development of new relationships between organisms and their environment; this would be accompanied by the discovery of novel trophic pathways and 
opportunities for species interaction, while biological structures reorganized and increased in complexity as new econiches were occupied (Dobretsov et al. 2007). Biological milestones such as the onset of endosymbiosis (Kiers and West 2015; Sagan 1966) and the rise of photosynthesis, the appearance of the first metazoans, the development of new trophic pathways like predation, and the exploration of new habitats mark further such transitions in ecological relationships, simultaneously increasing ecosystem stability and reducing conflict for shared resources by multiplying and subdividing econiches. Adaptive radiation and increasing physiological complexity allowed greater creativity in resource allocation and in ecological relationships, and organisms found fewer direct competitors as their trophic pathways and lifestyles became more specialized and unique. This trend remains true even for sessile communities like plants (Fort 2020), which reduce inevitable long-term competition for water, soil, and sunlight resources through strategies such as complementary adaptation (e.g. one plant type evolves to dwell in the shade of a taller sun-loving plant species, or an assemblage of herbs develop roots of different lengths and morphologies to minimize competition for soil and water). The relationship between these land plants thus tends toward a sort of "cooperative" development based on reciprocal growth styles and mutually subordinated ecological niches, attained through complementary adaptation and diversification.

The main point here is that these manifold processes of increasing diversification and complexity lead directly to the reduction of conflict and to increases in the extent of stabilizing cooperative relationships across the biosphere, providing a basis for "fractal" ecosystem evolution from the first molecular symbiotic proto-ecosystems to the modern biosphere (Prosdocimi et al. 2020). This tendency manifests itself across biological history as organisms develop ever more complex and diverse ways of interacting with themselves through 
increasingly sophisticated physiology and anatomy, with their environment through niche construction, and with other organisms through more integrated trophic and ecological relationships, often based on complementary adaptation and mutual communication (Markos and Svorcova 2019).

Diversification is not just associated with decreasing conflict, but also with increasing opportunities for organisms to explore new ways to resolve conflict and more widely distribute resources, forming relationships of positive mutualism between species and individuals. Complementary adaptation during diversification may lead to symbiosis, while symbiosis and other mutualisms assist in turn with diversification and niche construction (Moran 2006; OdlingSmee et al. 2003; Fort 2020). Many researchers have contributed to a fuller understanding of symbiosis and other mutualisms in evolutionary biology (see for instance Maynard Smith and Szathmary 1995; Moran 2006; Douglas 2010; Guerrero and Berlanga 2016; de Vries et al. 2018; Kiers et al. 2011; Markos and Svorcova 2019; and others). From the beginning of the biosphere, where the process of "chemical symbiosis" may have been crucial in the origin of life itself (Prosdocimi et al. 2020), symbiosis on many levels has played a dynamic and fundamental role in driving adaptation, diversification, and ecological coevolution of organisms and communities, enabling them to explore new econiches as well as terminating insufficiently adaptive lineages (Moran 2006; Douglas 2010; Stachowicz 2011; Maynard Smith and Szathmary 1995). On a microscopic level, endosymbiosis and bacterial symbioses in the lives of prokaryotes and all eukaryotes are considered pivotal in understanding the evolution of life, as well as in the survival of many organisms (Stachowicz 2011; Douglas 2010; Markos and Svorcova 2019; Guerrero and Berlanga 2016; Moran 2006; Morris et al. 2011, 2012; Kiers and West 2015; Sagan 1966). On a macroscopic level the evidence for symbiosis with bacteria, mycorrhizal fungi, insects, and 
larger pollinators as crucial in the development of land plant life has been well documented (de Vries et al. 2018; Kiers et al. 2011; Moran 2006; Ramos and Schiestl 2019), while the evolution of ecological relationships among animals was also greatly aided by mutualisms such as symbiosis (Moran 2006; Maynard Smith and Szathmary 1995). Indeed, cooperative interactions among populations and organisms are generally recognized as ubiquitous and crucial to healthy ecosystem functioning; for a more detailed discussion of this, see for instance Bruno et al. (2003), among others.

Such cooperation is not limited to individual species-species interactions, however. Indeed, some maintain that ecosystems consist of communicating and mutually interacting communities with shared reserves of evolutionary "memory" or umwelt; in the view of S. Kauffman, biospheres are defined by and coevolve in synchrony with these constituent communities, which "co-construct" the biosphere itself as well as their cohabitants within it (Kauffman 2000; Markos and Svorcova 2019). Moreover, it has been argued that a hallmark of all living systems is the constant transfer and interpretation of information through shared channels of communication, whether transmitted through shared symbionts and niches, propagated stimuli and signaling (Markos and Svorcova 2019), metabolic interconnectivity (Guerrero and Berlanga 2016), or biogeochemical feedback mechanisms operating globally (Vermeij and Roopnarine 2013; Lovelock and Margulis 1974). Thus, all species on Earth may be treated as interdependent, mutually integrated into holobiotic and ecological systems, and communicative through various pathways (Markos and Svorcova 2019; Dobretsov et al. 2007), and these qualities are typical both of integrated ecosystems and of the intimate relationships between symbionts. This implies that all organisms share in a global integrated relationship, analogous to individual symbiosis. 
Various forms of this concept of "metasymbiosis", in which all communities and organisms are integrated into the biosphere in a macroscale symbiotic relationship, have been propounded for many years, particularly by researchers and thinkers such as Lovelock, Margulis, and Roughgarden, among others (see for instance Lovelock 1989; Lovelock and Margulis 1974; Vernadsky 1926; Dobretsov et al. 2007; Roughgarden 2009; Hine 2019). Though this "Gaia" concept has long been controversial (Tyrrell 2013), increasing diversity may be expected to widen the scope of any such ecological integration across the biosphere; increasing biodiversity provides more opportunities for organisms to explore mutually beneficial relationships with each other and with the ecosystem as a whole, as well as to develop ecological redundancies which are favorable for Black Queen cooperative evolution (Morris et al. 2012). At the same time, biodiversity is also enhanced by cooperation and other facilitative relationships (Fort 2020). Thus, it is here argued that these symbiotic and metasymbiotic relationships between organisms and the biosphere have evolved in positive feedback with increasing diversity and complexity over biological history, and that these manifest a cyclical evolutionary tendency towards increasing harmony between organisms and within ecosystems.

\section{Competition and Cooperation at Equilibrium in the Biosphere}

Through reviewing these tendencies in the evolution of the biosphere, this essay seeks to illustrate a preferential movement of ecosystems away from states of conflict towards the cooperation of all communities composing them. Ecosystems seek this cooperative stability primarily through symbiotic relationships (Maynard Smith and Szathmary 1995), diversification of species via niche construction (Odling-Smee et al. 2003) and complementary adaptation, and metasymbiotic integration of member organisms and communities; ancient and constantly maintained pathways of communication among living systems underlie all these processes 
(Markos and Svorcova 2019). Of course, no ecosystem can operate without competition; it can be argued that whenever one aspect of the ecosystem changes, even as a side effect of cooperative evolution, the propagated changes to the local community will create ecological instabilities, leading to inevitable competition in other sectors of the ecosystem. This intuitively aligns with Lotka-Volterra dynamic models of competition between and within populations (e.g. Ackland and Gallagher 2004; Hening and Nguyen 2018), as well as the rigorous quantitative models of evolutionary Niche Game Theory, which have predicted that inter-species competition and cooperation will coexist at equilibrium in every ecosystem, with the ratio between cooperation and competition determined by local factors via dilemma games (Fort 2008; Fort 2020). Nonetheless, there is no necessary contradiction between such analyses and the conclusion presented here; although competition continually appears and equilibrates in ecosystems, the above tendencies imply that these equilibria across the biosphere (and hence in every ecosystem composing it) will preferentially evolve toward an increasing ratio of cooperation over competition whenever possible, such that maximized cooperation between communities is ultimately favored as the most stable ecosystem configuration, here referred to as a long-term "cooperative equilibrium" state.

Why, however, should cooperation be favored over competition as biosphere evolution progresses? There are a number of possible reasons for this tendency; of these, perhaps the most obvious is the role cooperation plays in long-term ecological conflict resolution. The survival and evolution of all life depends on resolving conflict, either competitively (e.g. by winning contests for resources and escaping rivals) or by establishing cooperative resolution. Unresolved mutual conflict forms a feedback system which ultimately becomes destructive for all parties and destabilizes evolving ecosystems (Vermeij and Roopnarine 2013), and this unstable 
configuration must be resolved through either competitive means or cooperative ones.

However, even the most stable competitive relationships do not fully resolve conflicts, but rather regulate them; even if pure conflict evolves into stable competition or asymmetrical ecological relationships, the dynamics between the competitors are still based upon some degree of conflict and hence are liable to degenerate into further instability. An optimally stable ecological configuration may be attained through cooperative interactions, in which each species supports the others and helps stabilize the ecosystem, resolving conflicts through mutual adaption and communication. Indeed, cooperation and harmony among and within these living systems greatly enhances their stability and facilitates beneficial interactions with other such systems, providing a framework for further diversification, as is implied by research in biological complexity theory (Prosdocimi et al. 2020; Kauffman 2013). Cooperative relationships also allow the distribution of more net benefits and resources to a larger number of more diverse niche occupants than otherwise would be possible (Vermeij and Roopnarine 2013). Thus, cooperative relationships are more effective at maintaining ecosystem stability and species survival than competition, particularly over large timescales, and this may help explain the tendencies discussed above.

How do these concepts fit in with existing evolutionary theory? Further work in this area is needed, but there are suggestive indications of compatibility between the view of evolution presented here and a number of paradigms in evolutionary biology, particularly the dominant and complementary models of Phyletic Gradualism and Punctuated Equilibria (von Vaupel Klein 1994). In this view, the rate and type of conflict resolution may determine the mode of evolution in an ecosystem, determining the relative importance of Punctuated Equilibria or Phyletic Gradualism processes in restoring ecosystem stability. Rapid and highly competitive evolution 
in response to major ecological instabilities might be associated with extreme "competitive disequilibrium" conditions, and manifest Punctuated Equilibria-type processes (Eldredge and Gould 1972). On the other hand, a more cooperative ecosystem with high species diversity might be expected to evolve more slowly, in synchrony with changing environmental conditions, with results as expected under Phyletic Gradualism.

Ultimately, these tendencies toward increasing cooperation in the biosphere and in local ecological equilibria may help illuminate the relationship between "Red Queen" and "Black Queen" behavior in evolving ecosystems. In the view presented by Vermeij and Roopnarine, the Red Queen hypothesis assumes that constant quantities of ecological benefits are distributed via zero-sum competition, while in reality ecosystems manifest increasing interconnectivity and growth in resource production over history (Vermeij and Roopnarine 2013). Thus, Red Queen processes and the high levels of conflict leading to them would be unstable in the long term (Vermeij and Roopnarine 2013), typifying a state of competitive disequilibrium, in some cases volatile (as in extinction events or incursions of invasive species) and in other cases changing very slowly (as in Lotka-Volterra competition between coexisting species). Cooperative processes, whether associated with increasing biodiversity or with Black Queen reductive adaption (Morris et al. 2012), might conversely be associated with falling levels of conflict and increasingly stable interwoven ecosystems, associated with a long-term equilibrium state where the ratio of cooperation to competition in the biosphere approaches maximal cooperative stability. While this movement toward biosphere stability is interrupted sporadically by extinction events and invasions by foreign species, states of extreme conflict resulting from such events are not observed to last; these ecosystems typically move through a restorative and often competitive phase back towards a diverse, integrated, and relatively cooperative ecological 
configuration. Examples of these ecosystems, such as bacterial assemblages evolving dependencies through mutual adaptation (Morris et al. 2011; Harvey et al. 2014), ecosystems recovering from invasions of foreign species (Chisholm 2010), or uninhabited regions colonized by a small number of species adaptively radiating through rapid allopatric and hybrid speciation (Lamichhaney et al. 2018; Ekimova et al. 2019), imply that this pattern of niche exploration and complementary adaptation away from initial conflict does generally play out in the long term. (See also Vermeij and Roopnarine 2013 for a further discussion of extinction events and similar ecological instabilities, and their resolution). Thus, competitive stress may spontaneously drive ecosystems toward increasingly cooperative equilibrium states, but such cooperative frameworks are not observed to spontaneously move back toward states of conflict, just as natural processes spontaneously move toward equilibrium but prefer not to move away from it.

\section{Implications for Future Research}

In this view, the Red Queen and Black Queen hypotheses are complementary, and represent different stages in the evolutionary resolution of ecological conflict, where conflict (associated with Red Queen processes and competitive disequilibrium) drives a rapid shift towards increasing cooperative stability (including processes like Black Queen evolution), with the ecosystem and the biosphere ultimately approaching "cooperative equilibrium". If the argument presented here is accepted, perhaps this tendency toward increasing cooperation could be incorporated into future mathematical models in evolutionary biology, particularly as a longterm limit in the evolution of Fort equilibria (Fort 2020; Fort 2008) and other game theory models. Furthermore, this may also have implications for the perennial debate regarding the role of conflict in the evolution of life, between proponents of an evolutionary paradigm based on conflict between "selfish" genes and organisms (e.g. Dawkins 1976) and proponents of a more 
harmonious view of life and nature (e.g. Paley 1802; Roughgarden 2009; Billington 1968). If it is accepted that living systems tend to evolve away from conflict towards cooperation, then this might imply that ecological harmony plays a more fundamental role in long-term biosphere evolution than is often believed. While conflict undeniably plays a crucial role in evolution, perhaps it chiefly acts as an unstable "disequilibrium" process, driving evolution towards a more diverse and cooperative biosphere rather than driving a perennial Red Queen "arms race" (van Valen 1973; Vermeij and Roopnarine 2013). In any case, further research in this area is required, including further developments of Niche Game Theory modelling and more observations of natural ecosystems to test existing theoretical constructs (Agrawal et al. 2007).

\section{Conclusion}

In summary, it is here argued that cooperation plays a key and increasing role in biosphere evolution, primarily through symbiosis among organisms and metasymbiosis among communities, attained through the coevolution of increasing diversity and complexity across ecological history. It is also maintained that biological evolution is controlled by the resolution of conflict between organisms, and that this conflict resolution is preferentially attained through mutual adaption and communication between organisms and their environment over time, as the relative benefits and stability imparted by cooperative integration are greater than those given by competitive ecological relationships. Taking these broad tendencies in consideration with Niche Game Theory, it may be maintained that, while competition and cooperation coexist at equilibrium in ecosystems (Fort 2008), such ecological equilibria shift preferentially toward higher ratios of cooperation to competition in the long term, directing the biosphere toward cooperative equilibrium as a limit in biosphere evolution. 
This idea could potentially resolve the tension between the Red Queen and Black Queen hypotheses, as it understands them as defining different aspects of the same evolutionary approach towards greater ecological harmony on multiple levels, and it could also bear mathematical and philosophical implications for future models of ecosystem evolution. In an analogical sense, these processes are comparable to a rhythmic or musical "melody" of cyclical competitive tension continually approaching cooperative resolution, just as the notes and themes composing a symphony move through cycles of tension towards a climax or harmonious consummation. This preferential evolutionary shift from conflict toward increasing cooperation and integration in a diverse and complex biosphere may help unveil the underlying mystery and beauty in nature, and better define the relationship between conflict and cooperation in the context of this overarching harmony. Whether or not these ideas are found acceptable by the scientific community, on this one truth at least evolutionary biologists of all persuasions may agree; that all life is mysterious and beautiful, and in the variety of its myriad forms and interactions there is ever more to explore. 


\section{References:}

1. Ackland, GJ, and Gallagher, ID (2004) Stabilization of large generalized Lotka-Volterra foodwebs by evolutionary feedback. Phys. Rev. Lett., 93(15), 158701.1-158701.4. https://doi.org/10.1103/PhysRevLett.93.158701

2. Agrawal, AA, Ackerly, DD, Adler, F, Arnold, AE, Cáceres, C, Doak, DF, Post, E, Hudson, PJ, Maron, J, Mooney, KA, Power, M, Schemske, D, Stachowicz, J, Strauss, S, Turner, MG, Werner, E (2007) Filling Key Gaps in Population and Community Ecology. Front. in Ecol. and the Environ., 5(3):145-152. https://doi.org/10.1890/15409295(2007)5[145:FKGIPA]2.0.CO

3. Billington, E (1968) Understanding Ecology. Frederick Warne and Co., New York, NY.

4. Bonner, JT (1988) The Evolution of Complexity by Means of Natural Selection, Princeton University Press, Princeton, NJ.

5. Bruno, JF, Stachowicz, JJ, and Bertness, MD (2003) Inclusion of facilitation into ecological theory. Trends in Ecol. and Evol, 18(3):119-125. https://doi.org/10.1016/s0169-5347(02)00045-9

6. Chisholm, RA (2010) Exponential growth in invasion biology. (Book review of Davis, MA (2009) Invasion Biology, Oxford University Press, Oxford, UK.) BioScience, 60(4):314-315. https://doi.org/10.1525/bio.2010.60.4.10

7. Dawkins, R (1976) The Selfish Gene. Oxford University Press, Oxford, UK.

8. de Vries, S, de Vries, J, von Dahlen, J K, Gould, SB, Archibald, JM, Rose, LE, \& Slamovits, CH (2018) On plant defense signaling networks and early land plant 
evolution. Comm. and Integr. Biol, 11(3):1-14.

https://doi.org/10.1080/19420889.2018.1486168

9. Dobretsov, N, Kolchanov, N, Rozanov, A, Zavarzin, G (2007) Biosphere Origin and Evolution. Springer Publishing, Boston, MA. https://doi.org/10.1007/978-0-387$\underline{68656-1}$

10. Douglas, AE (2010) The Symbiotic Habit. Princeton University Press, Princeton, NJ.

11. Ekimova, I, Valdes, A, Chichvarkhin, A, Antokhina, T, Lindsay, T, Schepetov, D (2019) Diet-driven ecological radiation and allopatric speciation result in high species diversity in a temperate-cold water marine genus Dendronotus (Gastropoda: Nudibranchia). Mol. Phylogenetics and Evol, 141:106609 https://doi.org/10.1016/j.ympev.2019.106609

12. Ekstig, B (2015) Complexity, Natural Selection and the Evolution of Life and Humans. Found. Sci., 20(2):175-187. https://doi.org/10.1007/s10699-014-9358-y

13. Eldredge, N, and Gould, SJ (1972) Punctuated equilibria: an alternative to phyletic gradualism. In: Schopf, TJM, ed., Models in Paleobiology, 82-115. Freeman, Cooper and Cy, San Francisco, CA.

14. Fort, H (2020) Combining niche and game theories to address interspecific cooperation in ecological communities. Community Ecol., 21(1):13-24. https://doi.org/10.1007/s42974-020-00006-7

15. Fort, H (2008) A minimal model for the evolution of cooperation through evolving heterogeneous games. Europhys. Lett., 81(4):48008. https://doi.org/10.1209/0295$\underline{5075 / 81 / 48008}$ 
16. Guerrero, R, Berlanga, M (2016) From the Cell to the Ecosystem: The Physiological Evolution of Symbiosis. Evol. Biol, 43:543-552. https://doi.org/10.1007/s11692-015$\underline{9360-5}$

17. Harvey, BP, Al-Janabi, B, Broszeit, S, Cioffi, R, Kumar, A, Aranguren-Gassis, et al. (2014) Evolution of marine organisms under climate change at different levels of biological organisation. Water, 6(11):3545-3574. https://doi.org/10.3390/w6113545

18. Hening, A, \& Nguyen, DH (2018) Persistence in Stochastic Lotka-Volterra Food Chains with Intraspecific Competition. Bull. of Math. Biol., 80(10):2527-2560. https://doi.org/10.1007/s11538-018-0468-5

19. Hine, R (2019) ed. "Gaia Hypothesis" from A Dictionary of Biology ( $8^{\text {th }}$ edn.), Oxford University Press, Oxford, UK. https://doi.org/10.1093/acref/9780198821489.001.0001

20. Kauffman, S (2000) Investigations. Oxford University Press, New York, NY.

21. Kauffman, S (2013) Evolution beyond Newton, Darwin, and entailing law: the origin of complexity in the evolving biosphere. In Lineweaver, CH, Davies, P, and Ruse, M (2013) ed. Complexity and the Arrow of Time, Cambridge University Press, Cambridge, UK. ProQuest Ebook Central, http://ebookcentral.proquest.com/lib/purdue/detail.action?docID=1303669

22. Kiers, E, Duhamel, M, Beesetty, Y, Mensah, J, Franken, O, Verbruggen, E, et al. (2011) Reciprocal Rewards Stabilize Cooperation in the Mycorrhizal Symbiosis. Science, 333(6044):880-882. https://doi.org/10.1126/science.1208473

23. Kiers, ET, and West, SA (2015) Evolving New Organisms via Symbiosis. Science (AAAS) 348(6233):392-94. https://doi.org/10.1126/science.aaa9605 
24. Kropotkin, PA (1902) Mutual aid; a factor of evolution, by P. Kropotkin. William Heinemann, London, England. https://hdl.handle.net/2027/dul1.ark:/13960/t1bk6qd1v

25. Lamichhaney, S, Han, F, Webster, M, Andersson, L, Grant, B, Grant, P (2018) Rapid hybrid speciation of Darwin's finches. Science, 359(6372):224-227. https://doi.org/10.1126/science.aao4593

26. Lineweaver, CH, Davies, P, \& Ruse, M (2013) ed. Complexity and the arrow of time. Cambridge University Press, Cambridge, UK. ProQuest Ebook Central, https://ebookcentral.proquest.com/lib/purdue/reader.action?docID=1303669

27. Lovelock, JE (1989) Geophysiology, the science of Gaia. Rev. Geophys. (1985), 27(2):215-222. https://doi.org/10.1029/RG027i002p00215

28. Lovelock, JE, \& Margulis, L (1974) Atmospheric homeostasis by and for the biosphere: The gaia hypothesis. Tellus, 26(1-2):2-10. https://doi.org/10.3402/tellusa.v26i1-2.9731

29. Markos, A, and Svorcova, J (2019) Epigenetic Processes and the Evolution of Life. CRC Press, Boca Raton, FL.

30. Maynard Smith, J and Szathmary, E (1995) The Major Transitions in Evolution. Oxford University Press, Oxford, England.

31. McGhee, GR, Muller, GB, Wagner, GP, Pradeau, T, Schafer, K (2011) Convergent Evolution: Limited Forms Most Beautiful. MIT Press, Cambridge, MA. ProQuest Ebook Central, https://ebookcentral.proquest.com/lib/purdue/detail.action?docID=3339309

32. Mitteldorf, JJ (2012) Adaptive aging in the context of evolutionary theory. Biochem. (Moscow), 77(7):716-725. https://doi.org/10.1134/s0006297912070036 
33. Moran, NA (2006) Symbiosis. Curr. Biol, 16(20):R866-R871. https://doi.org/10.1016/j.cub.2006.09.019

34. Morris, JJ, Lenski, RE, Zinser, ER (2012) The Black Queen Hypothesis: evolution of dependencies through adaptive gene loss. mBio 3(2):e00036-12. https://doi.org/10.1128/mbio.00036-12

35. Morris, JJ, Johnson, ZI, Szul, MJ, Keller, M, \& Zinser, ER (2011) Dependence of the Cyanobacterium Prochlorococcus on Hydrogen Peroxide Scavenging Microbes for Growth at the Ocean's Surface. PloS One, 6(2):e16805. https://doi.org/10.1371/journal.pone.0016805

36. Odling-Smee, GJ, Laland, KN, and Feldman, MW (2003) Niche Construction: The neglected process in evolution. Princeton University Press, Princeton, NJ.

37. Onori, L, \& Visconti, G (2012) The GAIA theory: From Lovelock to Margulis. From a homeostatic to a cognitive autopoietic worldview. Atti Della Accad. Naz. Dei Lincei. Rend. Lincei. Sci. Fis. E Nat., 23(4):375-386. https://doi.org/10.1007/s12210-012-0187$\underline{\mathrm{Z}}$

38. Paley, W (1802) Natural Theology. Wilks \& Taylor, London, UK.

39. Prosdocimi, F, José, MV \& de Farias, ST (2020) The Theory of Chemical Symbiosis: A Margulian View for the Emergence of Biological Systems (Origin of Life). Acta Biotheor. https://doi.org/10.1007/s10441-020-09388-7

40. Ramos, SE, and Schiestl, FP (2019) Rapid plant evolution driven by the interaction of pollination and herbivory. Science, 364(6436):193-196. https://doi.org/10.1126/science.aav6962 
41. Roughgarden, J (2009) The Genial Gene: Deconstructing Darwinian Selfishness. University of California Press, Berkeley, CA.

42. Sachs, JL, \& Hollowell, AC (2012) The origins of cooperative bacterial communities. mBio, 3(3):e00099-12. https://doi.org/10.1128/mBio.00099-12

43. Sagan, L (1966) On the origin of mitosing cells. J Theor Biol, 14(3):225-274. https://doi.org/10.1016/0022-5193(67)90079-3

44. Stachowicz, JJ (2011) Symbiosis (book review of Douglas, AE (2010) The Symbiotic Habit. Princeton University Press, Princeton, NJ.). BioScience, 61(4):326-327. https://doi.org/10.1525/bio.2011.61.4.16

45. Tyrrell, T (2013) On Gaia : A Critical Investigation of the Relationship Between Life and Earth. Princeton University Press, ProQuest Ebook Central, https://ebookcentral.proquest.com/lib/purdue/detail.action?docID=1184270

46. Van Valen, L (1973) A new evolutionary law. Evol. Theor. 1(1):1-30.

47. Vermeij, GJ, \& Roopnarine, PD (2013) Reining in the Red Queen: The dynamics of adaptation and extinction reexamined. Paleobiol., 39(4):560-575. https://doi.org/10.1666/13009

48. Vernadsky, VI (1926) The Biosphere. Nauchnoe khimiko-technicheskoye izdatel'stvo, Leningrad, Russia (in Russian).

49. von Vaupel Klein, JC (1994) Punctuated equilibria and phyletic gradualism: Even partners can be good friends. Acta Biotheor 42:15-48. https://doi.org/10.1007/BF00706838 